\title{
Certificación, Recertificación y Acreditación en Medicina Familiar y Comunitaria en Iberoamérica
}

\author{
Certification, Recertification and Accreditation in Family and Community Medicine in \\ Iberoamerica

\section{Certificação, Recertificação e Acreditação em Medicina de Família e Comunidade na Ibero-América}

Haydee Cabrera Reyes. Grupo Iberoamericano de Certificación, Recertificación y Acreditación en Medicina Familiar; Sociedad Campechana de Especialistas en Medicina Familiar. A. C. México. cabrehay@ hotmail.com (Autora correspondiente)

Diana Yuruhán Mohrbach. Grupo Iberoamericano de Certificación, Recertificación y Acreditación en Medicina Familiar; Sociedad Paraguaya de Medicina Familiar; Universidad Católica de Nuestra Señora de la Asunción. Paraguay. dyuruhan@ hotmail.es (Autora correspondiente) Patricia Vargas. Grupo Iberoamericano de Certificación, Recertificación y Acreditación en Medicina Familiar Facultad de Medicina; Universidad de Chile (UCh); Sociedad Científica de Medicina Familiar. Santiago, Chile. elizavargas@u.uchile.cl

Nancy del R. López Vásquez. Grupo Iberoamericano de Certificación, Recertificación y Acreditación en Medicina Familiar; Universidad de Chile (UCh); Sociedad Científica de Medicina Familiar y General. Santiago, Chile. nancylopez@med.uchile.cl Johanna Montalvo Vázquez. Grupo Iberoamericano de Certificación, Recertificación y Acreditación en Medicina Familiar. Sociedad Ecuatoriana de Medicina Familiar; Unidad de Medicina Familiar Hospital UTPL. Ecuador. johanna.montalvo@ hotmail.com

\section{Grupo de Trabajo}

Carlos Icaza. (Costa Rica), Diana Yuruhán Mohrbach. (Paraguay), Gerardo Ramírez Sandoval. (México), Gladys Sandoval. (Paraguay), Haydee Cabrera Reyes(México), Johanna Montalvo Vázquez. Montalvo J. (Ecuador), José Luis León. (Costa Rica), Karina Roselli. (Uruguay), Nancy del R. López Vásquez. (Chile), Patricia Vargas. (Chile), Xavier Astudillo Romero. (Ecuador) y Yossadara Luna Téllez. (México)

\section{Resumen}

Objetivo: Conocer el estado de la Certificación, Recertificación y Acreditación en los países de la región de Iberoamérica como seguimiento a la primera encuesta realizada en la V Cumbre Iberoamericana de Medicina Familiar en 2014 en Quito, Ecuador. Métodos: Diseño transversal descriptivo. La población estuvo conformada por diez países: Ecuador, Perú, Chile, Venezuela, México, Brasil, Paraguay, Colombia, Puerto Rico y Costa Rica. A los presidentes de las Asociaciones de Medicina Familiar, se les envió por e-mail una encuesta estructurada de doce preguntas acerca de la Certificación, Recertificación y Acreditación; la respuesta se recibió por la misma vía. Se efectuó un análisis estadístico descriptivo, frecuencias simples y relativas. Resultados: Las Sociedades Científicas participan en los procesos de Certificación, Recertificación y Acreditación en los países en los cuáles están instalados. La certificación se realizó en $60 \%$ de los países encuestados, este proceso fue voluntario en $40 \%$. La Recertificación está instalada y es operativa en $30 \%$ de los países participantes, este proceso es voluntario en $80 \%$ y lo ejecutan diferentes organismos, entre los cuales están las sociedades científicas, actuando solas o como parte de un equipo evaluador. De estos países, $50 \%$ de los países realizan la Acreditación de los programas de medicina familiar a través de las Universidades. Conclusiones: La Certificación todavía no se instaura en algunos países de lberoamérica, es voluntaria. Un menor porcentaje de países tienen implementado el proceso de Recertificación, el cual también es voluntario en su mayoría. Apenas la mitad de los países efectúan el proceso de Acreditación de las Unidades Formadoras.

Cómo citar: Cabrera HR, Yuruhán DM, Vargas P, López NV, Montalvo JV. Certificación, Recertificación y Acreditación en Medicina Familiar y Comunitaria en Iberoamérica. Rev Bras Med Fam Comunidade. 2016;11(Suppl 2):55-63. http://dx.doi.org/10.5712/rbmfc11(0)1386

\section{Palabras clave:}

Certificación

Recertificación

Acreditación

Medicina Familiar

y Comunitaria

\section{Financiación:}

ninguna declarada.

Aprobación ética:

La investigación fue

considerada una investigación

sin riesgo. Los autores declaran

que los procedimientos

seguidos se realizaron de acuerdo a las normas éticas de la Asociación Médica Mundial y la Declaración de Helsinki.

Conflicto de intereses: ninguna declarada.

Procedencia y revisión por pares: revisado por pares.

Recibido el: 15/08/2016.

Aceptado el: 15/09/2016. 


\begin{abstract}
Objective: To determine the status of the Certification, Recertification and Accreditation in the countries of the region of Latin America as a follow up to the first survey in the V Ibero-American Summit Family Medicine in 2014 inQuito, Ecuador. Methods: Cross-sectional descriptive. The population consisted of 10 countries: Ecuador, Peru, Chile, Venezuela, Mexico, Brazil, Paraguay, Colombia, Puerto Rico and Costa Rica. To the presidents of associations of family medicine, they were sent by e-mail a structured survey of twelve questions about certification, recertification and accreditation; the response was received by the same route. Descriptive statistics and simple and relative frequencies was made. Results: Certification is carried out in $60 \%$ of the surveyed countries; this process is voluntary in $40 \%$ and performs scientific societies. The recertification is installed and is operational in $30 \%$ of the participating countries, this process is voluntary in $80 \%$ and running different organisms among which are scientific societies. $50 \%$ of countries conduct the accreditation of family medicine programs through universities. Conclusions: Certification not yet instituted in some countries in Latin America, continues to be voluntary, and only half of the countries make the accreditation process. Therefore, we must work more in each of the countries in the region to achieve certification, recertification and accreditation to ensure the quality of specialists in Family Medicine.
\end{abstract}

\section{Resumo}

Objetivo: Conhecer a situação da Certificação, Recertificação e Acreditação nos países da região da Ibero-Americana como seguimento do primeiro levantamento realizado na V Cúpula lbero-Americana de Medicina de Família em 2014 em Quito, Equador. Métodos: desenho transversal descritivo. A população do estudo foi composta por dez países: Equador, Peru, Chile, Venezuela, México, Brasil, Paraguai, Colômbia, Porto Rico e Costa Rica. Se enviou por email um questionário estruturado de doze perguntas sobre Certificação, Recertificação e Acreditação; a resposta foi recebida pela mesma via. Foi realizada uma análise estatística descritiva, com frequências simples e relativas. Resultados: As sociedades científicas envolvidas participam nos processos de Certificação, Recertificação e Acreditação nos países em que são implementados. A certificação foi realizada em $60 \%$ dos países pesquisados, este processo foi voluntário em $40 \%$. Recertificação é implementada e está ativa em $30 \%$ dos países participantes, este processo é voluntário para $80 \%$ e são realizadas por diferentes organizações, entre as quais sociedades científicas, atuando isoladamente ou como parte de uma equipe de avaliação. Destes países, $50 \%$ procedem à acreditação de programas de medicina de família através de universidades. Conclusões: A Certificação ainda não está estabelecida em alguns países da lbero-América, é voluntária. Uma porcentagem menor de países implementaram o processo de recertificação, o qual é voluntário em sua maioria. Apenas metade dos países realizam o processo de acreditação das Unidades Formadoras.
Keywords:

Certification

Recertification

Accreditation

Family and Community

Medicine

Palavras-chave:

Certificação

Recertificação

Acreditação

Medicina de Família

e Comunidade

\section{Introducción}

Los procesos de cambio que se presentan con la globalización económica, establecen requerimientos de calidad al ejercicio de la medicina que incluyen la certificación de una práctica profesional óptima y competitiva en beneficio de la población. ${ }^{1}$ Uno de estos aspectos, es la mejora de la calidad en la atención médica, donde se han incluido estrategias como la certificación y recertificación de manera a tratar de garantizarla. ${ }^{2}$ En este punto, es esencial definir a qué se le llama calidad de la atención médica. Este concepto varía en diferentes épocas y sociedades; sinembargo, el concepto propuesto por Donabedianes integrador yglobalizador para aceptarlo como definición de ella: "Calidad es el grado en que los medios másdeseables se utilizan para alcanzar las mayores mejoras posibles". ${ }^{3}$ Ruelas señala que "Ia calidad es una combinación de beneficios, riesgos y costos, en donde de lo que se trata es deofrecer los mayores beneficios con los menores riesgos posibles y a un costo razonable". Así mismo, Aguirre-Gas, dice que "la calidad de la atención médica es otorgar atención oportuna al usuario, conforme los conocimientos médicos y principios éticos vigentes, con satisfacción de las necesidades de salud y de las expectativas del usuario, del prestador de servicios y de la institución". 4-6

Derivado de lo anterior, la certificación profesional es un proceso que garantiza estándares técnicos y socialmente aceptables de calidad de cuidados para los usuarios de los servicios de salud. Dominan un cuerpo de conocimientos y/o experiencias relevantes en un ámbito de trabajo asistencial, para otorgarle el certificado correspondiente; ${ }^{7}$ por lo tanto, es de suma importancia que exista la certificación de los especialistas en Medicina Familiar en todos los países de Iberoamérica. ${ }^{8}$ La certificación en Medicina Familiar puede entenderse como un proceso de validación que permite garantizar a la sociedad que el profesional posee los conocimientos y competencias necesarias y suficientes 
para brindar atención integral y continua al individuo y su familia con elevados estándares de Calidad, basados en los principios y prácticas de la Especialidad..$^{9,10}$ Los médicos de familia deben ser los responsables y protagonistas activos en definir, implementar y administrar los procesos de certificación en las instituciones que certifiquen profesionales. ${ }^{2}$ A pesar de que la certificación es un estándar de calidad que todos los médicos de familia deben tener; no obstante, algunos de los países de la región Iberoamericana aún no la han implementado. En otros, como Estados Unidos, es de carácter voluntario ${ }^{10}$ y en pocos, como en México, la certificación es obligatoria (2011), plasmada en el artículo 83 de la Ley General de Salud. ${ }^{11}$

Por lo tanto, como consecuencia de los avances tecnológicos que ocurren permanentemente en las ciencias médicas, así como la transición epidemiológica y demográfica que tiene lugar constantemente en todas las sociedades, los especialistas en medicina familiar están obligados a renovar y actualizar periódicamente sus conocimientos. Por tanto, es necesario comprobar la vigencia de su competencia profesional a través de la recertificación. Esto es posible a través de la adquisición de nuevas competencias emergentes, dado el crecimiento del conocimiento y la complejidad de la tarea del médico de familia, dentro de los sistemas de salud. ${ }^{7,8,10}$ En Medicina Familiar, la recertificación pretende mantener el compromiso de atención médica con elevados estándares de calidad, tanto al individuo como a su familia, de acuerdo a los avances de la medicina familiar y a las demandas de salud de la población. ${ }^{9}$

Para complementar estos procesos, es necesario que se desarrolle un sistema de acreditación de los programas de Residencias en Medicina Familiar. La acreditación debe entenderse como un proceso mediante el cual una organización es capaz de medir la calidad de sus servicios o productos, y el rendimiento de los mismos frente a estándares reconocidos a nivel nacional e internacional. ${ }^{12}$ Así también, la acreditación es un proceso de constatación del cumplimiento de los programas por parte de las Instituciones de Educación Superior, de los requisitos para la formación de los profesionales en las diferentes especialidades de la medicina, en concordancia con el estado del arte de la medicina en el mundo y las necesidades de las personas y la comunidad. ${ }^{13}$

En la Declaración de Margarita, se establece que cada país debe ser responsable de establecer los requisitos mínimos de acreditación de Residencias en Medicina Familiar. Su objetivo es garantizar un perfil básico de competencias profesionales en todas y cada una de las unidades formadoras de especialistas de los países, para lo cual se sugiere considerar los siguientes aspectos: nombre de la especialidad, perfil profesional, tiempo de duración, requisitos de ingreso, requisitos de permanencia, requisitos de egreso, estructura curricular, requisitos para los docentes, características de las sedes académicas de Medicina Familiar y características de las sedes académicas hospitalarias de apoyo. ${ }^{8}$ Esto significa que el proceso de acreditación representa un enorme esfuerzo, en el que las instituciones educativas de salud y civiles deben participar para elevar los niveles de calidad que presta el médico de familia. ${ }^{14}$

La Carta de Quito en la V Cumbre Iberoamericana de Medicina Familiar recomienda: "Reconocer que la Certificación y Acreditación profesional en Medicina Familiar y Comunitaria permite el fortalecimiento de la calidad de atención en el tiempo y estimula la actualización del profesional."

Por todo lo anterior, un conjunto de especialistas en Medicina Familiar conformaron un grupo Iberoamericano de trabajo compuesto por ocho países. En un primer momento construye y recoge información a partir de una encuesta elaborada por este comité de expertos. Con la información obtenida se realizó un análisis FODA (fortalezas, oportunidades, debilidades y amenazas) y se compararon los niveles de avance de los procesos de certificación y acreditación de la especialidad en Medicina Familiar de Ecuador, Venezuela, Bolivia, México, Argentina, Paraguay, Brasil y Chile. Los resultados se presentaron en el 2014 durante la V Cumbre Iberoamericana de Medicina Familiar en Quito, Ecuador. ${ }^{15}$

El presente estudio constituye el segundo momento del grupo de trabajo con el objetivo de conocer el estado de la Certificación, Recertificación y Acreditación en los países de la región de Iberoamérica como seguimiento a la primera encuesta.

\section{Métodos}

Se efectuó un estudio de diseño transversal, descriptivo en diez países de la región Iberoamericana WONCA-CIMF (World Organization of FamilyDoctors - Confederación Iberoamericana de Medicina Familiar): Brasil, Chile, Colombia, 
Costa Rica, Ecuador, México, Paraguay, Perú, Puerto Rico y Venezuela, durante el período de noviembre de 2015 a marzo de 2016; que contaban con Sociedades Científicas o Asociaciones de Medicina Familiar. A los presidentes o integrantes del comité directivo de las asociaciones o sociedades, se envía por medios electrónicos (e-mail) una encuesta estructurada de 12 preguntas acerca de las variables certificación, recertificación y acreditación en Medicina Familiar y Comunitaria; la respuesta se recibió por la misma vía.

Cabe destacar que la encuesta se envió a 20 países; de ellos, solo los mencionados con anterioridad respondieron. No se recibió contestación de Argentina, Bolivia, Uruguay, Cuba, España, El Salvador, Panamá, Portugal, Honduras y República Dominicana.

El análisis de los datos se efectuó mediante estadística descriptiva y frecuencias simples y relativas con el Programa Estadístico SPSS v.21.

\section{Resultados}

De los países a los que se envió la encuesta, sólo la mitad de ellos respondió (10/20). De los encuestados, 40\% se desempeñan como directivos de la Sociedad, $30 \%$ como presidente y $30 \%$ es miembro de la Sociedad de Medicina Familiar, de entidad acreditadora y director de Posgrado.

En la figura 1 se muestran los datos de la pregunta ¿El proceso de Certificación en Medicina Familiar se realiza en su país?, Se observa que 40\% (4 países) aún no realiza este proceso. Es importante señalar que en sólo dos países el proceso de Certificación es obligatorio, uno ellos es México en donde es obligatorio desde 2011 y el otro es Venezuela mientras que para cuatro de los países de la región CIMF la certificación es voluntaria (Figura 2).

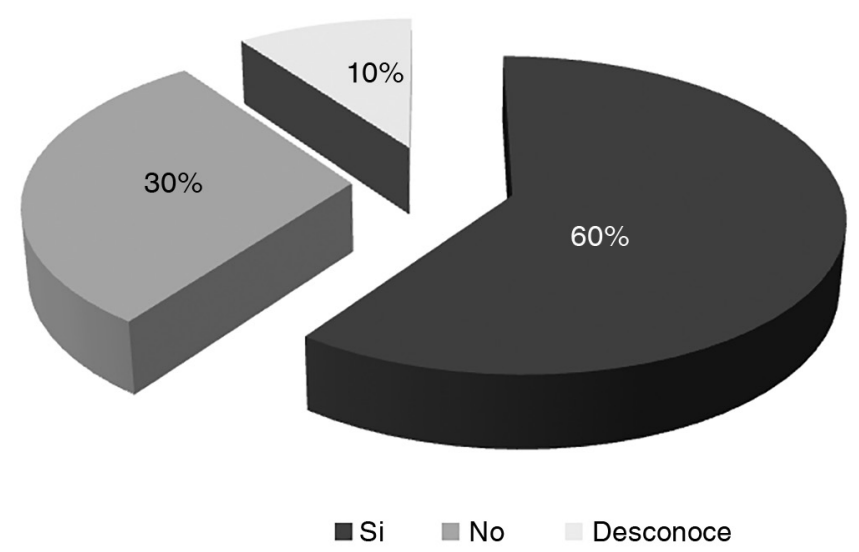

Figura 1. Realización del Proceso de Certificación en el País.

Fuente: Encuesta GICRAMF* F 2015-2016.

* Nota: Grupo Iberoamericano de Certificación, Recertificación y Acreditación en Medicina Familiar.

En la mayoría (6/10) de los países, los organismos certificadores de los especialistas en Medicina Familiar la ejecutan las Sociedades Científicas, Colegio de Médicos y sólo en México la certificación la realiza el Consejo Mexicano de Certificación (Tabla 1).

La recertificación está instalada y es operativa en el 30\% de los países participantes, este proceso es voluntario en el $80 \%$ y lo ejecutan diferentes organismos entre los cuales están las sociedades científicas, el consejo de certificación y organismos públicos y privados.

Respecto al proceso de acreditación de los programas de formación de la especialidad en Medicina Familiar, se encontró que en la mitad de los países encuestados (5/10) está instaurada la acreditación (Figura 3). 


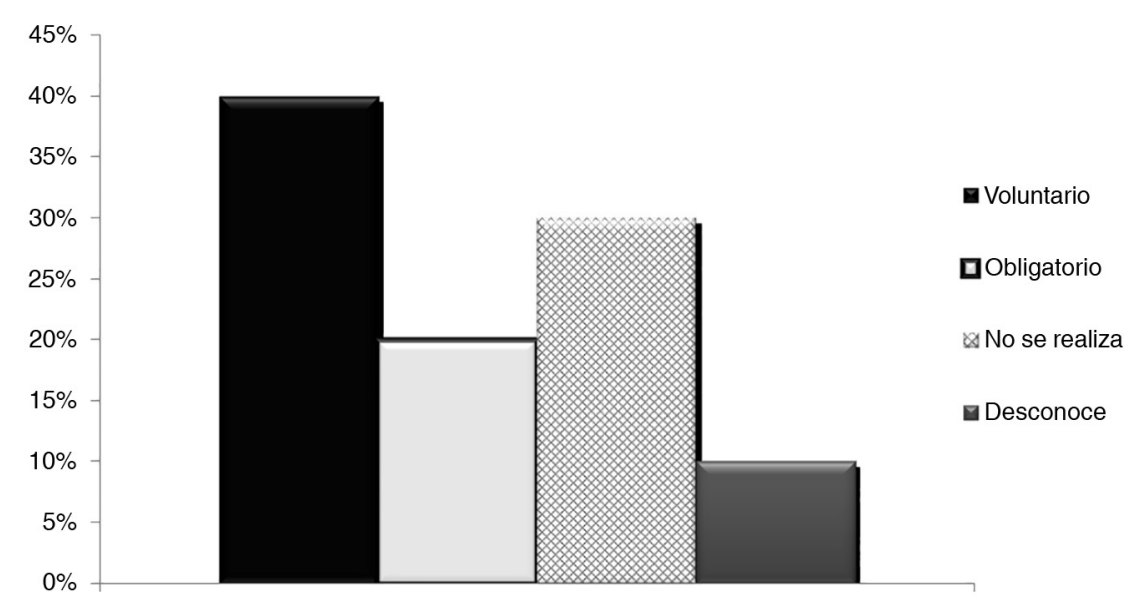

Figura 2. Obligatoriedad el proceso de Certificación.

Fuente: Encuesta GICRAMF* - Informantes Países CIMF 2015-2016.

Tabla 1. Organismos certificadores de los Médicos Familiares por países CIMF

\begin{tabular}{|c|c|c|c|c|}
\hline \multirow[b]{2}{*}{ País } & \multicolumn{4}{|c|}{ Organismos Certificadores } \\
\hline & $\begin{array}{c}\text { Sociedad Científica y } \\
\text { Colegio Médico }\end{array}$ & Universidades & Ministerio de Salud & Consejo de Certificación \\
\hline Brasil & $x$ & & & \\
\hline Chile & $x$ & $x$ & & \\
\hline Costa Rica & $x$ & & & \\
\hline \multicolumn{5}{|l|}{ Colombia } \\
\hline \multicolumn{5}{|l|}{ Ecuador } \\
\hline México & & & & $x$ \\
\hline Paraguay & $x$ & & & \\
\hline \multicolumn{5}{|l|}{ Perú } \\
\hline \multicolumn{5}{|l|}{ Puerto Rico } \\
\hline Venezuela & $x$ & $x$ & & \\
\hline
\end{tabular}

Nota: Ecuador no cuenta con certificación. Fuente: Encuesta GICRAMF 2015-2016.

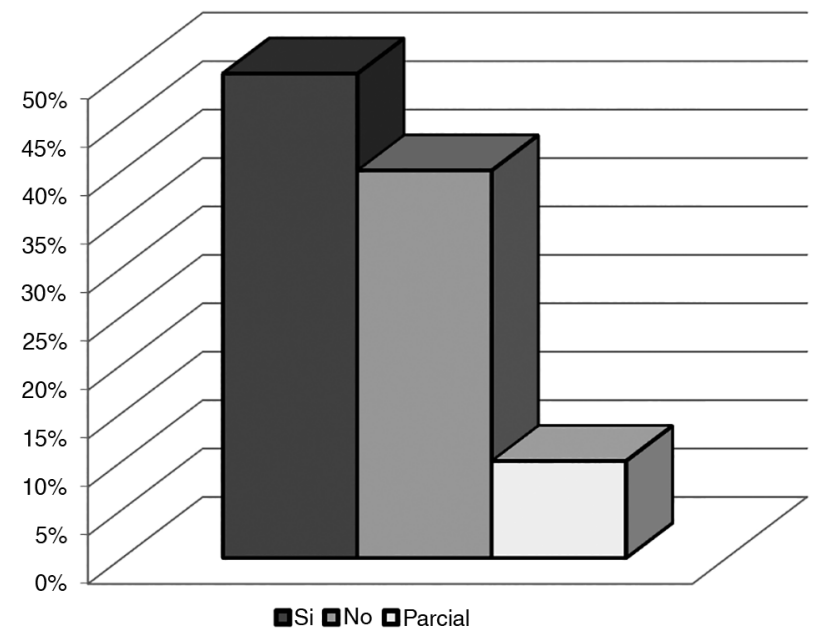

Figura 3. Realización del proceso de Acreditación en el País. Fuente: Encuesta GICRAMF 2015-2016. 
Las principales entidades acreditadoras son las universidades (3/10), seguidas por los organismos públicos y privados (4) y las sociedades científicas. En la figura 4 se muestra cómo es el proceso de acreditación en los países. A nivel nacional, los programas de formación del médico familiar la mitad de los países tienen diferencias (50\%) e iguales en 40\%; así mismo, los programas varían poco (2/10).

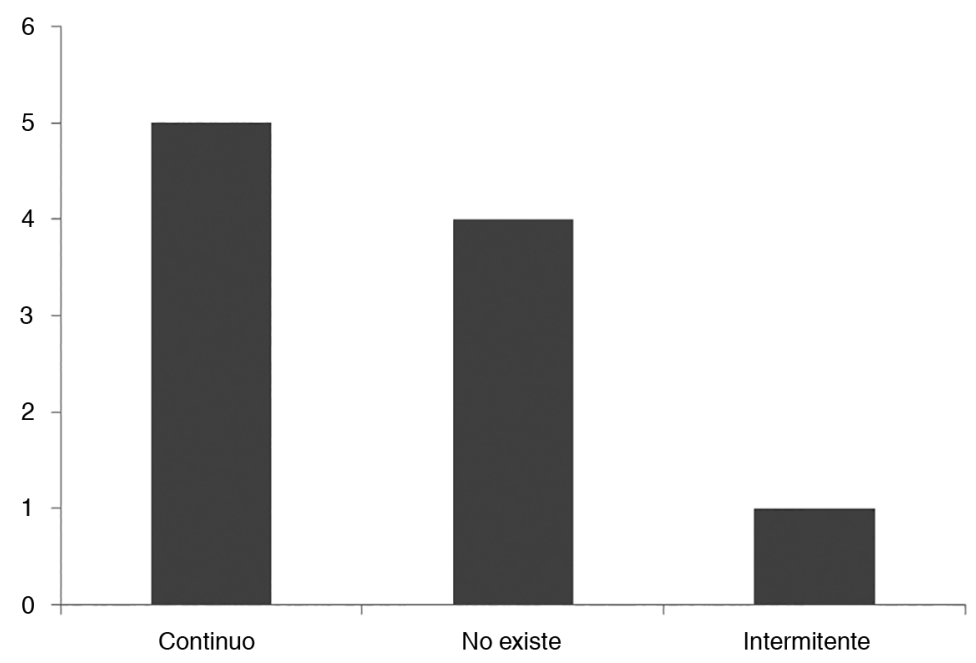

Figura 4. Continuidad del proceso de Acreditación en el País. Fuente: Encuesta GICRAMF 2015-2016.

\section{Discusión}

\section{Resumen de los principales hallazgos del estudio}

La certificación todavía no se instaura en algunos países de Iberoamérica, en otros continúa siendo voluntario; un porcentaje aún menor de países tienen implementado el proceso de recertificación, el cual también es voluntario y apenas la mitad de los países efectúan el proceso de acreditación. Por lo tanto, se debe trabajar más en la sensibilización en cada uno de los países de la región para lograr que la certificación, recertificación y acreditación en Medicina Familiar se realice en Iberoamérica para garantizar la calidad de los programas de formación en la especialidad, y por ende de los especialistas en Medicina Familiar, lo que repercutirá en la mejoría de la calidad de las atención de los pacientes y sus familias.

Uno de los principales hallazgos de este estudio es que las sociedades científicas participan en la mayoría de los procesos de certificación y recertificación de especialistas en Medicina Familiar, con respecto a la acreditación de los programas de formación, las universidades son las principales entidades acreditadoras.

\section{Fortalezas y limitaciones del estudio.}

\section{Fortalezas}

Una de las fortalezas del estudio se refiere a que existe un mayor compromiso a nivel de las sociedades en lo referente a la certificación, recertificación y acreditación, así mismo, se observa el compromiso de las Sociedades que contestaron la encuesta de fortalecer los procesos de certificación y recertificación. Otra de ellas, es que permitió identificar a las instituciones u organismos que certifican la especialidad en medicina familiar de los países de Iberoamérica. 
El proceso de Acreditación de los programas de formación en Medicina Familiar es continuo en la mitad de los países encuestados, lo que pudiera significar que la calidad en los programas de formación de los especialistas en Medicina Familiar comienza a ser una preocupación. Además el estudio, permite conocer a las instituciones u organismos que acreditan los programas de formación de la especialidad de MF, evalúa los aspectos de acreditación de los programas, al igual reconoce si estos programas de acreditación están acreditados en los países de lberoamérica.

Se considera que la principal fortaleza, reside en que la encuesta fue dirigida a los especialistas en Medicina Familiar involucrados en los procesos de certificación, recertificación y acreditación, por lo que su opinión es con conocimiento de causa.

\section{Limitaciones}

Las limitaciones más importantes encontradas fueron la dificultad en la comunicación y obtención de los datos tanto para su envío y repuesta de la encuesta con los países integrantes del CIMF (Confederación Iberoamericana de Medicina Familiar).

También es una debilidad el hecho de que la encuesta no fue validada por otros expertos, únicamente por los médicos de familia del grupo Iberoamericano, y la encuesta no fue enviada de manera conjunta a todos los países de la región. La encuesta estuvo claramente dirigida a los temas de interés del grupo y la evaluación de la encuesta fue realizada por algunos integrantes del grupo y fue difícil dirigir la discusión. Finalmente, no existen elementos que ayuden a un estudio y evaluación más cualitativa en los temas de certificación, recertificación y acreditación de los programas de formación de Especialistas en Medicina Familiar.

\section{Comparación con la literatura existente}

A pesar de que la certificación es un estándar buscado desde hace mucho tiempo, llama la atención la gran diversidad de interpretaciones que se dan a este término en los diferentes países. Para algunos, la certificación es aquella que se obtiene al concluir los estudios de posgrado en una residencia médica y que corresponde a la aprobación de las evaluaciones correspondientes que permitan acreditar al médico para el ejercicio de la profesión. En otros países se denomina certificados a los médicos que al concluir la residencia en un programa formal, no aprueban las evaluaciones correspondientes, y por ese motivo deben someterse a una evaluación extraordinaria, que les permita acreditar su competencia como especialista ante la sociedad. En otros se le llama certificación a la acreditación de médicos generales como especialistas en Medicina Familiar, a través de la aprobación de algunos cursos, prácticas clínicas, etc., y que son evaluados por algún comité académico. ${ }^{14}$

El hecho de que en $60 \%$ de los países encuestados tengan instaurado un proceso de certificación, nos demuestra que los especialistas en Medicina Familiar son conscientes de la necesidad de garantizar la calidad de su oferta médica; sin embargo, los diferentes modelos existentes de estos procesos pueden ser considerados como punitivos, por lo que es imprescindible una buena pedagogía para explicar de una manera muy clara qué es este proceso y los beneficios que tiene para que no sea visto como un examen, sino como un método que "mejora la competencia de los profesionales". ${ }^{16}$

\section{Implicaciones para la investigación en el área y/o la práctica de los profesionales}

Existen varias implicaciones de la investigación, derivadas de la importancia otorgada a estos procesos de Certificación, Recertificación y Acreditación por la comunidad misma y los Sistemas de Salud de cada país. Destacamos las más significativas: ¿Cuán importantes son estos procesos en los países?, ¿Qué entidades regulan estos procesos?, ¿Cómo utilizar los resultados obtenidos después del análisis efectuado en beneficio de los profesionales y la población? El mayor desafío que se nos presenta, es realizar un modelo de estos procesos para Iberoamérica tomando en cuenta el análisis de los resultados de esta y futuras investigaciones. 
En la práctica profesional es indispensable que los especialistas en Medicina Familiar deban tener una evaluación. Esta debe ser en dos momentos: al terminar su proceso de formación (Certificación) y durante su carrera (Recertificación), de esta manera estaríamos garantizando que el profesional presenta y mantiene las competencias de un especialista en Medicina Familiar. De igual forma, dada la diversidad de programas de formación que existe en Iberoamérica en Medicina Familiar, es necesario que estos sean acreditados para certificar su concordancia con el perfil profesional del Médico(a) Familiar. En esta virtud es necesario tener un diagnóstico situacional actualizado de estos tres procesos en Iberoamérica siendo el punto de generación de futuras investigaciones en el campo.

Estos procesos deben responder a la necesidad del Estado es por ello que en los mismos debe conformarse un equipo de trabajo entre los organismos gubernamentales legalmente establecidos, la academia y las Sociedades Científicas de Médicos Familiares a fin de que se garantice un sistema de evaluación integral con el Perfil del Médico Familiar.

La Certificación y Recertificacióndeben constituirse en procesos voluntarios en un principio de manera a lograr que los especialistas valoren su importancia y se sientan jerarquizados en el ejercicio profesional. Sin embargo, los Sistemas Nacionales de Salud de cada país deben garantizar las competencias de sus profesionales en Medicina Familiar a través de la instauración formal de estos procesos con un mecanismo de evaluación por pares sin considerarlos como punitivos sino como la oportunidad de validar las competencias adquiridas a lo largo de su formación y de su profesión, y que puedan ir adaptándose a los cambios científicos ocurridos en el tiempo.

Las Sociedades científicas deben tomar un rol activo y un compromiso ofreciendo a los Médicos de Familia oportunidades accesibles de capacitación a través de Educación Médica Continua, llegando a los asociados a través de medios virtuales o presenciales.

\section{Conclusiones}

La certificación todavía no se instaura en algunos países de Iberoamérica, es voluntaria. Un menor porcentaje de países tienen implementado el proceso de recertificación, el cual también es voluntario en su mayoría. Apenas la mitad de los países efectúan el proceso de acreditación. Por lo tanto, se debe trabajar más en cada uno de los países de la región para lograr que la certificación, recertificación y acreditación en Medicina Familiar se realice en Iberoamérica para garantizar la calidad de los programas de formación en medicina familiar, y por ende de los especialistas en Medicina Familiar, lo que repercutirá en los pacientes y sus familias.

Considerando que ocasionalmente existen distintos programas de formación dentro de un mismo país, se hace necesario estandarizar los mecanismos de certificación y los de acreditación. Esto constituye un desafío para las Sociedades y Asociaciones científicas, al igual que para el CIMF conseguir una matriz básica de evaluación aplicable a los diferentes países y que refleje las competencias a ser obtenidas. Seguramente será necesario trabajar de una manera coordinada y continua en cada uno de los países de la región para lograr que los procesos de certificación, y recertificación de la especialidad y la acreditación de los programas de medicina familiar se realicen en forma continua en Iberoamérica con el objetivo de garantizar la calidad de los programas de formación en medicina familiar, y de los especialistas en Medicina Familiar.

\section{Referencias}

1. Comité Normativo Nacional de Medicina General. Boletín Atención Familiar. 1998;5(3-4):10-13.

2. Brennan TA; Horwitz, RI; Duffy, D; Cassel, CK; Goode, LD; Lipner, RS. The role of Physician Specialty Board Certification Status in the Quality Movement. JAMA. 2004;29:1038-43.

3. González LE, Gallardo EG. Calidad de la Atención Médica: la diferencia entre la vida o la muerte. Revista Digital Universitaria. 2012;13(8):1-5. Disponible en: http://www.revista.unam.mx/vol.13/num8/art81/index.html

4. Ruelas E. Calidad en Salud... ¿Pleonasmo necesario? Conferencia en el Primer Foro de Calidad en Salud. Calimed 1998; 4(3-4): $55-60$ 
5. Fernández H, Sotelo G. La CONAMED y la calidad de la atención médica. MedicinaUniversitaria 2000;2(8):232-38.

6. Aguirre-Gas H. Administración de la calidad de la atención médica. Revista Médica del Instituto Mexicano del seguro Social. 1997;35(4):257-64.

7. Reglamento de Certificación de Especialidades, Ley de Educación Superior en Chile. Decreto N 57 de 2007 . https://www. supersalud.gob.cl/normativa/.../articles-4799_recurso_1.pdf

8. Declaración de Margarita. Boletín Atención Familiar. 2003;10(6):3.

9. Estatutos Vigentes del Consejo mexicano de certificación en Medicina Familiar. Disponible en: https://www.consejomedfam. org. $m x$

10. Fernández-Ortega MA, Cuevas M, Alderete JA, Barell AE. Convergencia de procesos de certificación y recertificación de especialistas en Estados Unidos y México. MedIntMéx. 2016 mayo; 32(3):263-269.

11. Diario Oficial de la Federación. DOF 0 1/09/2011. México. Disponible en: http://www.dof.gob.mx/nota_detallephpcodigo?5207454

12. Reglamento APICE: Acreditación de Programas de Especialidades Médicas. Disponible en: http://www.apicechile.cl/site/

13. Jiménez D. Beneficios de la certificación y acreditación en la educación. Revista Coepestgo. 2013;3(8):1-6. Disponible en: http://www.revistacoepesgto. mx/revistacoepes8/beneficios-de-la-certificacion-y-acreditacion-en-la-educacion

14. Fernández Ortega MA, Yañez Puig EJ. La certificación y recertificación Médica en México. Atención Familiar. 2004;11(6):9-12.

15. Vargas P, Cabrera H, Montalvo J, López NR, Yuruhán D. Análisis comparativo de la certificación, recertificación y acreditación en Latinoamérica para la especialidad en Medicina Familiar y Comunitaria. RevBrasFamComunidade. 2016;12(Suppl 1):61-7.

16. OMC. La recertificación supone un beneficio para los médicos y los pacientes y redunda en un SNS más eficiente, según expertos. 2015. Disponible en: http://www.cgcom.es/noticias/2015/10/15_10_15_37_congreso_semergen 